\title{
Third order effect of postionization population redistribution in strong field
}

\author{
Xiangxu Mu, ${ }^{1,{ }^{*}}$ Ming Zhang, ${ }^{1, *}$ Hanwei Yang, ${ }^{1}$ Min Li $\odot,{ }^{2}$ Keyu Guo, ${ }^{2}$ Zijian Lü, ${ }^{1}$ \\ Jiechao Feng $\odot,{ }^{1}$ Haitan $\mathrm{Xu},{ }^{3,4, \dagger}$ Song Bin Zhang $\odot,{ }^{5, \star}$ and Zheng $\mathrm{Li}{ }^{1, \S}$ \\ ${ }^{1}$ State Key Laboratory for Mesoscopic Physics and Collaborative Innovation Center of Quantum Matter, \\ School of Physics, Peking University, Beijing 10087, China \\ ${ }^{2}$ Wuhan National Laboratory for Optoelectronics and School of Physics, \\ Huazhong University of Science and Technology, Wuhan 430074, China \\ ${ }^{3}$ Shenzhen Institute for Quantum Science and Engineering, Southern University of Science and Technology, Shenzhen 518055, China \\ ${ }^{4}$ School of Physical Sciences, University of Science and Technology of China, Hefei 230026, China \\ ${ }^{5}$ School of Physics and Information Technology, Shaanxi Normal University, Xi'an 710119, People's Republic of China
}

(Received 6 September 2021; accepted 3 November 2021; published 29 November 2021)

\begin{abstract}
In strong field ionization, the pump pulse not only photoionizes the molecule, but also drives efficient population exchanges between its ionic ground and excited states. In this study, we investigate the population dynamics after strong field molecular photoionization, using angular distribution of dissociative fragments. Our results reveal that the first and third order processes of the post-ionization population redistribution mechanism (PPRM) in the ion can be disentangled and classified by its angle-resolved kinetic energy release (KER) spectra. We demonstrate that the imprints of PPRM in the KER spectra can be used to determine the branching ratio of the population exchange pathways of different orders, by exploiting the pump-intensity-dependent variation of the spectra.
\end{abstract}

DOI: 10.1103/PhysRevResearch.3.043144

\section{INTRODUCTION}

The photoionization and dissociation of molecules induced by intense femtosecond laser pulse are fundamental physical processes of light-matter interactions $[1,2]$. The dissociation dynamics can be resolved in detail by delayed ultrashort probe laser pulses for a series of delays between the pump and probe laser pulses, where the pump pulse excites or ionizes the molecule and initiates the dissociation [3-13]. The ionization and dissociation of few-electron molecules, such as $\mathrm{H}_{2}$, have been exhaustively studied [14-26], e.g., Ref. [27] gives an excellent explanation on the intensity dependence of photofragments angular distributions of $\mathrm{H}_{2}^{+}$with barrier lowering (or bond softening) theory and nonadiabatic transition theory, and Ref. [28] provides a complete quantum modelization for angle-resolved kinetic energy release (KER) spectra of photofragments resulting from intense field dissociation with an assumption that transition dipole vector is parallel to the molecular axis.

However, the post-ionization electronic dynamics of manyelectron molecules does not have a coherent and comprehensive characterization of the underlying physical processes due

\footnotetext{
*These authors contributed equally to this work.

†xuht@sustech.edu.cn

‡song-bin.zhang@snnu.edu.cn

§ zheng.li@pku.edu.cn
}

Published by the American Physical Society under the terms of the Creative Commons Attribution 4.0 International license. Further distribution of this work must maintain attribution to the author(s) and the published article's title, journal citation, and DOI. to the complexity of the involved electronic states. For $\mathrm{N}_{2}^{+}$or $\mathrm{O}_{2}^{+}$, which has heavier core and larger energy difference, the coupling between the laser field and electronic states has more significant effect as the dissociation progresses.

In addition to the often adopted sudden ionization approximation, after the leaving of ionized electron from the molecular ion in a few hundreds of attoseconds [29-31], the electronic configurations between the ionic states can still be significantly modified within the duration of the ionization pulse, because the laser couples all the relevant ionic states allowed by symmetry. The ionization pulses with pulse duration of about $10 \mathrm{fs}$ can provide sufficient time for such laserdriven population dynamics. The resulting post-ionization population redistribution mechanism (PPRM) has proven to be important in many scenarios and applications of strong field physics [32-36]. As a prominent example, PPRM is especially crucial for the realization of population inversion in the $\mathrm{N}_{2}^{+}$air laser system driven by intense near infrared laser [37]. Quantitatively resolving the dynamics of PPRM is thus indispensable to devise new control mechanisms in these applications.

In this paper, we study the third order effect in postionization population redistribution which can be disentangled from the usual first order process using kinetic energy release spectra as a function of the angle between the molecular alignment axis and the linear polarization of the ionization pump pulse. We demonstrate that the pathways of population redistribution can be classified by the orders of transitions in the ionic states, and their branching ratios can be quantified by measuring angle dependent KER spectra of dissociative fragments of pre-aligned molecules at various pump intensities. The proposed theory can serve as a new recipe to 
analyze post-ionization population dynamics of molecules in strong laser field by means of angle-resolved dissociative ionization, e.g., using cold-target recoil-ion-momentum spectroscopy (COLTRIMS).

\section{THEORETICAL METHODS}

\section{A. Model for the kinetic energy release spectra}

Kinetic energy release spectra are crucial for the analysis of electronic dynamics and structural evolution of molecules in dissociative ionization experiments $[10,13,21,38]$. Because the tunneling ionization rate depends exponentially on the strength of laser electric field, the ionization dominantly occurs near the peak of the pump laser pulses. Supposing a linearly polarized ionization laser is used with polarization along the space-fixed $Z$ axis, and $\theta$ is the alignment angle between the molecular $z$ axis and the polarization of the ionization laser pulse. In our model, the dissociation of the ionic diatomic molecule is divided into two processes. First, ionization of a neutral molecule by an ultrashort intense pump laser happens mainly at the peak laser intensity and generates molecular ions in different excited electronic states within the Franck Condon approximation [33], and the $\theta$-dependent angular distributions of ionic molecules are described by MO-PPT theory [39], which correspond to the ionization rates $W_{v}(\theta)$ into electronic states $v$. Second, after the generation of molecular ions at peak laser intensity, the remaining half-falling pulse triggers the electronic state transition and dissociation of the molecular ions with alignment angle $\theta$ [37]. The dissociative state transferred from initial state $v$ dissociates and generates the kinetic energy spectra $D_{v}(\theta, \varepsilon)$ for the molecular ions, where $\varepsilon$ is the kinetic energy of the fragments. Thus, the total KER spectra can be constructed incoherently from the two processes described above as

$$
I_{\mathrm{KER}}(\theta, \varepsilon)=\sum_{v} W_{v}(\theta) D_{v}(\theta, \varepsilon)
$$

\section{B. Alignment-angle dependent dissociation}

To simulate the post-ionization population redistribution and dissociation dynamics induced by the ionization laser pulse, let us consider a diatomic molecular cation with alignment angle $\theta$ relative to the laser polarization axis, which is produced by ionization at the peak of the laser pulse [33]. The electronic states $|\nu\rangle$ and $|\lambda\rangle$ with potential energy curves (PEC) $V_{v}(r)$ and $V_{\lambda}(r)$ are coupled by the strong field of the laser pulse, when allowed by symmetry selection rules. Denote the distance between two atoms in diatomic molecule to be $r$, the Hamiltonian $\hat{H}_{\theta}$ of the molecular ion for a given $\theta$ can be written as

$$
\hat{H}_{\theta}=\hat{T}+\hat{H}_{\theta}^{\prime}-i \hat{W},
$$

where $\hat{T}$ is kinetic energy operator and $\hat{W}=\eta \Theta\left(r-r_{c}\right)(r-$ $\left.r_{c}\right)^{2}$ is the perturbed complex absorbing potential (CAP) [40]. $\Theta\left(r-r_{c}\right)$ is step function, where $r_{c}$ is the position of the flux plane and is chosen such that the potentials become constant for $r>r_{c}$. The potential and laser-molecule interaction
Hamiltonian $\hat{H}_{\theta}^{\prime}$ could be written as

$$
\hat{H}_{\theta}^{\prime}=\left(\begin{array}{ccc}
V_{\nu} & D_{v \lambda} & \cdots \\
D_{v \lambda}^{\dagger} & V_{\lambda} & \cdots \\
\vdots & \vdots & \ddots
\end{array}\right),
$$

where $\nu, \lambda$ label electronic states, $D_{\nu \lambda}=\mu_{\nu \lambda} E \cos \theta$ or $\mu_{\nu \lambda} E \sin \theta$ for allowed transitions with transition dipole moment $\mu_{\nu \lambda}$ being parallel or perpendicular to the molecular axis, respectively, and $E$ is electric field of the laser. The symmetries of the coupled electronic states play the role to determine the selection rules of the electronic transitions. Because the molecular axis has an angle $\theta$ relative to the polarization axis of the ionization laser, the nonzero perpendicular and parallel components of the transition dipoles drive the population transfer within the duration of the ionisation pulse. We solve the time-dependent Schrödinger equation (TDSE) of the nuclear wave packet, using the multiconfiguration timedependent Hartree (MCTDH) approach [40,41],

$$
i \frac{\partial}{\partial t}\left(\begin{array}{c}
\Psi_{v} \\
\Psi_{\lambda} \\
\vdots
\end{array}\right)_{\theta}=\hat{H}_{\theta}\left(\begin{array}{c}
\Psi_{v} \\
\Psi_{\lambda} \\
\vdots
\end{array}\right)_{\theta},
$$

where $\Psi_{v}$ is the nuclear wave functions in electronic eigenstate $|v\rangle$, and the atomic unit is used throughout the paper. The efficient implementation of the MCTDH algorithm is advantageous for the simulation involving various alignment angles and ionization laser intensities. The flux analysis is able to give the intensity of wave packet components of specific kinetic energies, which go through the flux plane at $r=r_{c}$. The population of dissociated wave packet is given by the $S$-matrix elements,

$$
\left|S_{v}\left(\varepsilon_{v}\right)\right|_{\theta}^{2}=\frac{d}{d t}{ }_{\theta}\left\langle\phi_{\nu}^{+}\left|\Theta\left(r-r_{c}\right)\right| \phi_{\nu}^{+}\right\rangle_{\theta},
$$

where $\phi_{\nu}^{+}$denotes the asymptotic wave function of electronic state $|v\rangle$. And the corresponding $S$-matrix elements could be calculated using CAP as [40,42]

$$
\begin{aligned}
\left|S_{\nu}\left(\varepsilon_{v}\right)\right|_{\theta}^{2}= & \frac{1}{\left|\Delta\left(\varepsilon_{v}\right)\right|^{2}} \iint d t d \tau_{\theta}\langle\Psi(t+\tau)|\hat{W}| \Psi(t)\rangle_{\theta} \\
& \times \exp (-i \varepsilon \tau),
\end{aligned}
$$

where the energy density function of the initial wave packet is

$$
\Delta\left(\varepsilon_{v}\right)=\langle\Psi(t=0)|\delta(\hat{H}-\varepsilon)| \Psi(t=0)\rangle,
$$

and $\varepsilon_{v}$ is the eigenenergy of continuum components of the outgoing wave packet. The alignment-angle-dependent and state-resolved kinetic energy release (KER) spectra of dissociative ionization fragments could be thus obtained by Eq. (6) for each electronic state $|v\rangle$ as $D_{v}(\theta, \varepsilon)=\left|S_{v}\left(\varepsilon_{v}\right)\right|_{\theta}^{2}$, where the asymptotic energy of each state $\varepsilon_{v}^{\text {as }}$ is subtracted, i.e., $\varepsilon=\varepsilon_{v}-\varepsilon_{v}^{\mathrm{as}}$.

\section{Alignment-angle dependent ionization}

Alignment-angle dependent tunneling ionization rates $W_{v}(\theta)$ from neutral to cationic state $|\nu\rangle$ is modelled with the 



FIG. 1. Rate of tunneling ionization from $\mathrm{N}_{2}$ to $\mathrm{N}_{2}^{+}$as a function of the alignment angle $\theta$ between the molecular axis and the laser polarization at peak laser intensities of $1.0 \times 10^{14} \mathrm{~W} / \mathrm{cm}^{2}$ (a)-(c) and $2.2 \times 10^{14} \mathrm{~W} / \mathrm{cm}^{2}$ (d)-(f). Black solid line, red dashed line, and blue point dashed line denote the ionization rates to the final states $X^{2} \Sigma_{g}^{+}, A^{2} \Pi_{u}$, and $B^{2} \Sigma_{u}^{+}$of $\mathrm{N}_{2}^{+}$cation.

MO-PPT theory $[39,43]$, which is written as

$$
\begin{aligned}
& W_{\nu, \mathrm{MO}-\mathrm{PPT}}(E, \omega, \theta) \\
& =\left(\frac{3 E}{\pi \kappa^{3}}\right)^{1 / 2} \sum_{m^{\prime}} \frac{B^{2}\left(m^{\prime}\right)}{2^{\left|m^{\prime}\right|}\left|m^{\prime}\right| !} \frac{A_{m^{\prime}}(\omega, \gamma)}{\kappa^{2 Z_{c} / \kappa-1}}\left(1+\gamma^{2}\right)^{\left|m^{\prime}\right| / 2+3 / 4} \\
& \quad \times\left(\frac{2 \kappa^{3}}{E}\right)^{2 Z_{c} / \kappa-\left|m^{\prime}\right|-1}\left(1+2 e^{-1} \gamma\right)^{-2 Z_{c} / \kappa} e^{\left[-\left(2 \kappa^{3} / 3 E\right) g(\gamma)\right]},
\end{aligned}
$$

where $E$ is the electric field strength, $\kappa=\sqrt{2 I_{p}}, I_{p}$ is the ionization potential of given molecular orbital, $\theta$ is the angle between molecular axis and laser polarization axis, and $\gamma=$ $\frac{\omega \sqrt{2 I_{p}}}{E}$ is the Keldysh parameter. Here, $\omega$ and $Z_{c}$ are the laser wave vector and the asymptotic charge seen by the electron. In this equation $g(\gamma)$ can be written as

$$
g(\gamma)=\frac{3}{2 \gamma}\left[\left(1+\frac{1}{2 \gamma^{2}}\right) \sinh ^{-1} \gamma-\frac{\sqrt{1+\gamma^{2}}}{2 \gamma}\right],
$$

and the coefficient $A_{m^{\prime}}(\omega, \gamma)$ can be written as

$$
\begin{aligned}
A_{m}(\omega, \gamma)= & \frac{4}{\sqrt{3 \pi}} \frac{1}{|m| !} \frac{\gamma^{2}}{1+\gamma^{2}} \\
& \times \sum_{\kappa \geqslant \nu}^{\infty} \mathrm{e}^{-\alpha(\gamma)(\kappa-\xi)} w_{m}(\sqrt{\beta(\gamma)(\kappa-\xi)}),
\end{aligned}
$$

where

$$
\begin{aligned}
w_{m}(x) & =\frac{x^{2|m|+1}}{2} \int_{0}^{1} \frac{\mathrm{e}^{-x^{2} t} t^{|m|}}{\sqrt{1-t}} d t, \\
\alpha(\gamma) & =2\left(\sinh ^{-1} \gamma-\frac{\gamma}{\sqrt{1+\gamma^{2}}}\right), \\
\beta(\gamma) & =\frac{2 \gamma}{\sqrt{1+\gamma^{2}}}, \quad \xi=\frac{I_{p}}{\omega}\left(1+\frac{1}{2 \gamma^{2}}\right), \\
\kappa & =\left\langle\frac{E_{0}}{\omega}+1\right\rangle+S, \quad(S=0,1, \ldots) .
\end{aligned}
$$

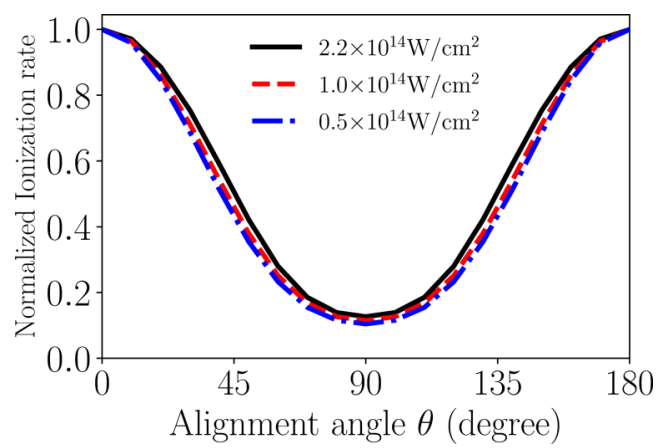

FIG. 2. Normalized distribution of total ionization rate to final states $X^{2} \Sigma_{g}^{+}, A^{2} \Pi_{u}$, and $B^{2} \Sigma_{u}^{+}$of $\mathrm{N}_{2}^{+}$as a function of alignment angle between molecular axis and laser polarization for different peak laser intensities. 


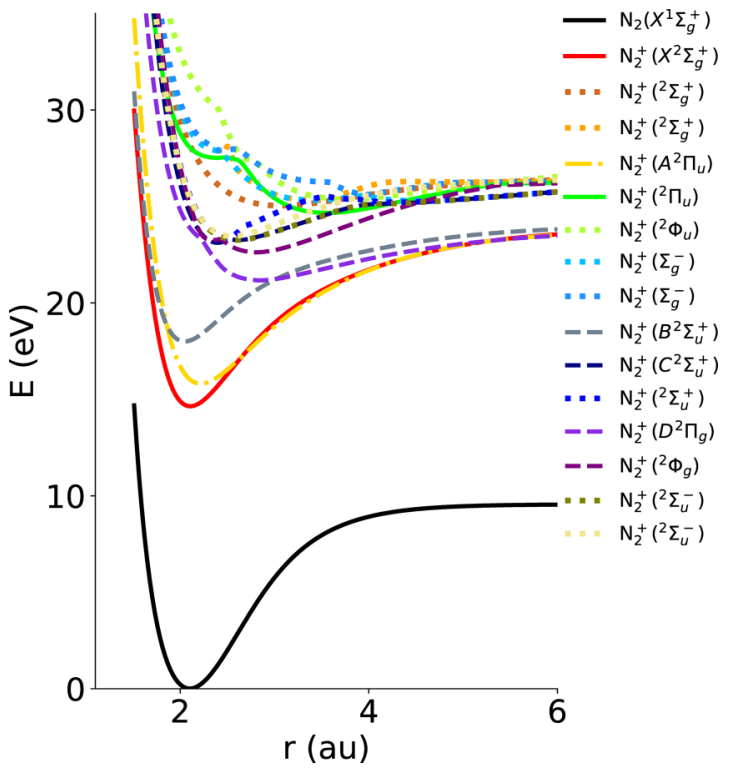

FIG. 3. The potential energy curves of electronic states of $\mathrm{N}_{2}^{+}$. Red solid line is for the cationic ground state $X^{2} \Sigma_{g}^{+}$, and green solid line is for the dissociative state ${ }^{2} \Pi_{u}$.

The symbol \langle\rangle indicates the integer part of the value inside. And for linear molecule,

$$
B\left(m^{\prime}\right)=\sum_{l} C_{l m} D_{m^{\prime}, m}^{l}(\theta) Q\left(l, m^{\prime}\right),
$$

where

$$
Q\left(l, m^{\prime}\right)=(-1)^{\left(m^{\prime}+\left|m^{\prime}\right|\right) / 2} \sqrt{\frac{(2 l+1)\left(l+\left|m^{\prime}\right|\right) !}{2\left(l-\left|m^{\prime}\right|\right) !}},
$$

$D_{m^{\prime}, m}^{l}(\theta)$ is rotation matrix, and $C_{l m}$ are the coefficients of molecular orbital given in $[30,31]$.

\section{RESULTS AND DISCUSSION}

In this section, we present the simulation of dissociative ionization of laser aligned $\mathrm{N}_{2}$ and $\mathrm{O}_{2}$ molecules, and the analysis of the post-ionization population dynamics of the molecular ions induced via PPRM by the ionization pulse. For all numerical applications discussed in this paper, we consider laser pulse with a fixed wavelength of $800 \mathrm{~nm}$ and a FWHM of $40 \mathrm{fs}$. The center of the pulse is chosen to be at $t=0 \mathrm{fs}$, where ionization mainly occurs [37]. The population redistribution and dissociation dynamics takes place in the falling edge of the laser pulse [33].

Via $a b$ initio quantum chemistry calculations by the MOLPRO package [44], we constructed accurate PECs of nitrogen molecule that consist of the three low-lying electronic states directly accessible by ionization $\left(X^{2} \Sigma_{g}^{+}, A^{2} \Pi_{u}\right.$, and $\left.B^{2} \Sigma_{u}^{+}\right)$, the dissociated state ${ }^{2} \Pi_{u}$, and intermediate states $B^{2} \Sigma_{u}^{+}$, $C^{2} \Sigma_{u}^{+}, D^{2} \Pi_{g}$, and ${ }^{2} \Phi_{g}$ of $\mathrm{N}_{2}^{+}$, and the ground state $X^{1} \Sigma_{g}^{+}$of $\mathrm{N}_{2}[45,46]$. For the oxygen molecule, we calculated the PECs for the low-lying electronic state $a^{4} \Pi_{u}$, dissociated state $f^{4} \Pi_{g}$ and intermediate states $b^{4} \Sigma_{g}^{-}, c^{4} \Sigma_{u}^{+},{ }^{4} \Sigma_{u}^{-},{ }^{4} \Sigma_{u}^{+}, f^{4} \Pi_{g}$, and ${ }^{4} \Sigma_{g}^{+}$of $\mathrm{O}_{2}^{+}$, and the ground state ${ }^{3} \Sigma_{g}^{-}$of neutral $\mathrm{O}_{2}$. Specifically, Dunning's aug-cc-pVTZ basis, active space including 14 valence orbitals of $\mathrm{N}$ atoms and $\mathrm{O}$ atoms were employed in the complete active space self-consistent-field (CASSCF) calculations. The calculated PECs are presented in Fig. 3 for $\mathrm{N}_{2}$ and $\mathrm{N}_{2}^{+}$and Fig. 7 for $\mathrm{O}_{2}$ and $\mathrm{O}_{2}^{+}$.

\section{A. Ionization and dissociation of $\mathrm{N}_{2}^{+}$cation}

Using the MO-PPT theory $[39,43]$, we calculated the rate of tunneling ionization from neutral $\mathrm{N}_{2}$ to $\mathrm{N}_{2}^{+}$cation as a function of alignment angle $\theta$ between molecular axis and laser polarization for two representative ionization laser peak intensities $I_{0}\left(1.0\right.$ and $\left.2.2 \times 10^{14} \mathrm{~W} / \mathrm{cm}^{2}\right)$. In the calculation of MO-PPT, $S$ is cut off at 35 to ensure the conversion of
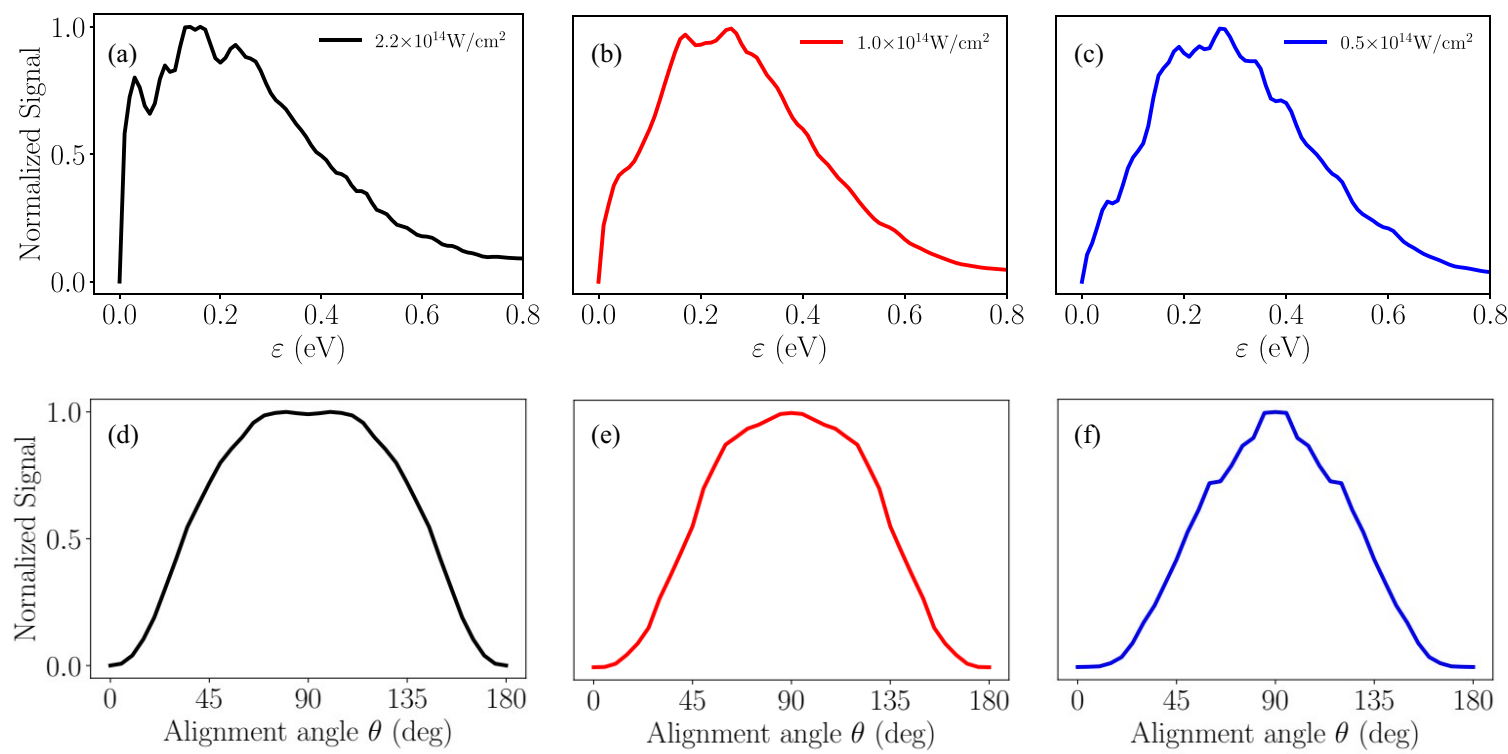

FIG. 4. Alignment-angle-integrated energy distribution (upper panel) and energy-integrated alignment angle dependence (lower panel) of normalized dissociation yields $D(\theta, \varepsilon)$ of $\mathrm{N}+\mathrm{N}^{+}$fragments. From left to right columns, the peak intensity of laser is $2.2 \times 10^{14} \mathrm{~W} / \mathrm{cm}^{2}$, $1.0 \times 10^{14} \mathrm{~W} / \mathrm{cm}^{2}$, and $0.5 \times 10^{14} \mathrm{~W} / \mathrm{cm}^{2}$, respectively. 

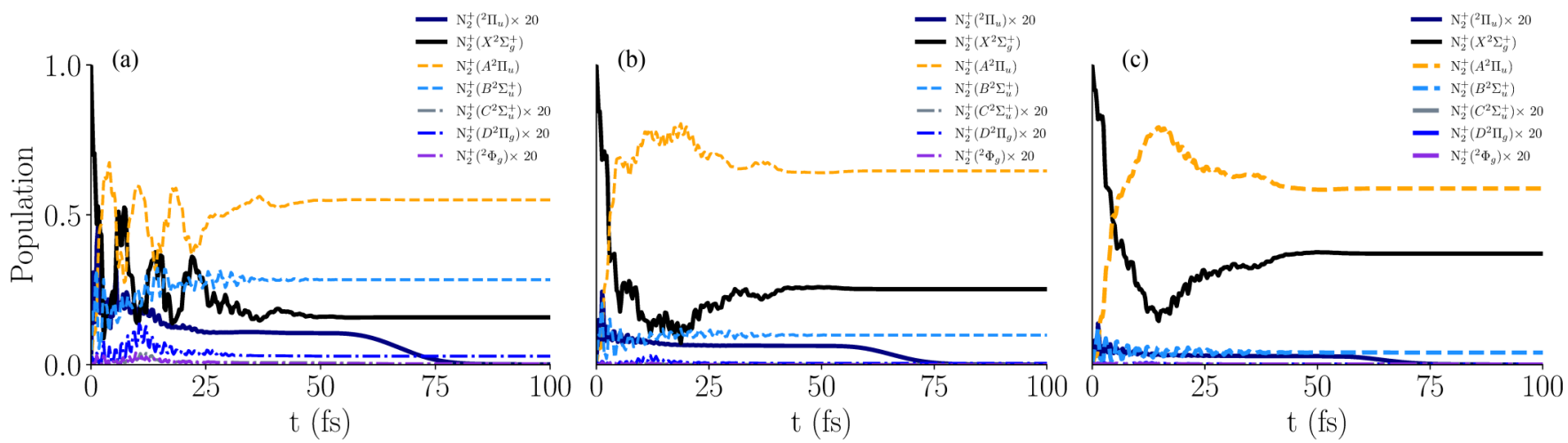

FIG. 5. The temporal population evolution of ${ }^{2} \Pi_{u}$ and intermediate states $B^{2} \Sigma_{u}^{+}, C^{2} \Sigma_{u}^{+}, D^{2} \Pi_{g},{ }^{2} \Phi_{g}$ of $\mathrm{N}_{2}^{+}$at peak laser intensities of $2.2 \times 10^{14} \mathrm{~W} / \mathrm{cm}^{2}, 1.0 \times 10^{14} \mathrm{~W} / \mathrm{cm}^{2}$, and $0.5 \times 10^{14} \mathrm{~W} / \mathrm{cm}^{2}$, respectively, and at alignment angle $\theta=60$ degrees.

ionization rate. And the alignment-angle-dependent ionization rate is shown in Fig. 1. The ionization rate of the final state $X^{2} \Sigma_{g}^{+}$and $B^{2} \Sigma_{u}^{+}$maximizes when molecular axis is parallel to laser polarization and minimizes when perpendicular, while ionization rate of final state $A^{2} \Pi_{u}$ maximizes at perpendicular alignment and minimizes at parallel alignment [31].

As shown in Fig. 2, the angular dependence of the ionization rate under different laser intensities by summing over states $X^{2} \Sigma_{g}^{+}, A^{2} \Pi_{u}$, and $B^{2} \Sigma_{u}^{+}$shows that the intensity does not affect the total angular distribution of ionization rate, and after the ionization the $X^{2} \Sigma_{g}^{+}$state is mostly populated.

Because of fast decoherence induced by the outgoing photoelectron in the sub-fs time scale [29], the coherence between the $X^{2} \Sigma_{g}^{+}, A^{2} \Pi_{u}$, and $B^{2} \Sigma_{u}^{+}$states are neglected in our model. And for the dissociation dynamics, the contribution from initial states $A^{2} \Pi_{u}$ and $B^{2} \Sigma_{u}^{+}$is negligible. Because from the MO-PPT calculation, even for the peak field intensity of $2.2 \times 10^{14} \mathrm{~W} / \mathrm{cm}^{2}$, ionization rate from neutral $\mathrm{N}_{2}$ to cationic $X^{2} \Sigma_{g}^{+}$state is two orders of magnitude higher than that to $A^{2} \Pi_{u}$ and $B^{2} \Sigma_{u}^{+}$.

For the $\mathrm{N}_{2}^{+}$cation, $X^{2} \Sigma_{g}^{+}, A^{2} \Pi_{u}, B^{2} \Sigma_{u}^{+}, D^{2} \Pi_{g},{ }^{2} \Phi_{g}$ are bound states and ${ }^{2} \Pi_{u}$ is a dissociative state, which is crossed by another state ${ }^{2} \Phi_{u}$ at $r=2.64$ a.u. (see Fig. 3). The predissociative $C^{2} \Sigma_{u}^{+}$state shows long lifetime of several nanoseconds [47], and its contribution to the KER spectra is negligibly small comparing to the direct dissociation channel in ${ }^{2} \Phi_{u}$ state, as confirmed by the MCTDH simulation. The

TABLE I. The first and third order pathways from $X^{2} \Sigma_{g}^{+}$to ${ }^{2} \Pi_{u}$ and the dependence of transition amplitude on electric field strength and alignment angle. $\mu_{\perp}$ denotes transition dipole along the direction perpendicular to the molecular axis and $\mu_{\|}$denotes transition dipole along the parallel direction.

\begin{tabular}{lc}
\hline \hline Channel & $(\theta, E)$-dependence \\
\hline${ }^{2} \Pi_{u} \stackrel{\mu_{\perp}}{\longleftarrow} X^{2} \Sigma_{g}^{+}$ & $E \sin \theta$ \\
${ }^{2} \Pi_{u} \stackrel{\mu_{\|}}{\longleftarrow} D^{2} \Pi_{g} \stackrel{\mu_{\perp}}{\longleftarrow} B^{2} \Sigma_{u}^{+} \stackrel{\mu_{\|}}{\longleftarrow} X^{2} \Sigma_{g}^{+}$ & $E^{3} \cos ^{2} \theta \sin \theta$ \\
${ }^{2} \Pi_{u} \stackrel{\mu_{\|}}{\longleftarrow}{ }^{2} \Phi_{g} \stackrel{\mu_{\perp}}{\longleftarrow} B^{2} \Sigma_{u}^{+} \stackrel{\mu_{\|}}{\longleftarrow} X^{2} \Sigma_{g}^{+}$ & $E^{3} \cos ^{2} \theta \sin \theta$ \\
${ }^{2} \Pi_{u} \stackrel{\mu_{\|}}{\longleftarrow} D^{2} \Pi_{g} \stackrel{\mu_{\perp}}{\longleftarrow} C^{2} \Sigma_{u}^{+} \stackrel{\mu_{\|}}{\longleftarrow} X^{2} \Sigma_{g}^{+}$ & $E^{3} \cos ^{2} \theta \sin \theta$ \\
${ }^{2} \Pi_{u} \stackrel{\mu_{\|}}{\longleftarrow}{ }^{2} \Phi_{g} \stackrel{\mu_{\perp}}{\longleftarrow} C^{2} \Sigma_{u}^{+} \stackrel{\mu_{\|}}{\longleftarrow} X^{2} \Sigma_{g}^{+}$ & $E^{3} \cos ^{2} \theta \sin \theta$ \\
\hline \hline
\end{tabular}

time-dependent wave functions in Eq. (4) are obtained by using the multiconfiguration time-dependent Hartree (MCTDH) method [40,41]. $\eta=0.03$ a.u. and $r_{0}=10$ a.u are used for CAP to calculate the photodissociation by flux analysis [42]. 512 sine-DVR basis functions are used for the $r$ grid [48]. In the simulation we include all the possible alignment angles of the molecular axis relative to the linear polarization of the ionization laser.

In the following sections, we investigate angle-resolved ionization rate of nitrogen molecule and the angle-resolved KER spectra $D(\theta, \varepsilon)$ of dissociated fragments, as well as their dependence on peak intensity of the ionization pulse, and they together provide the route towards disentangling the different pathways of population redistribution. In Fig. 4, we present the alignment-angle-integrated dissociation $D(\theta, \varepsilon)$ spectra for peak laser intensities of $2.2 \times$ $10^{14} \mathrm{~W} / \mathrm{cm}^{2}, \quad 1.0 \times 10^{14} \mathrm{~W} / \mathrm{cm}^{2}$, and $0.5 \times 10^{14} \mathrm{~W} / \mathrm{cm}^{2}$, respectively.

As demonstrated in Eq. (1), the final KER intensity depends on both the ionization rate and dissociation rate. In our MCTDH calculation from equally populated initial condition, the ratio of dissociation yields between $X^{2} \Sigma_{g}^{+}, A^{2} \Pi_{u}$, and $B^{2} \Sigma_{u}^{+}$states is $764: 75: 221$, which are of similar magnitude. However, multiplying the branching ratio of tunneling ionization, the ratio of dissociation yields from initial state $X^{2} \Sigma_{g}^{+}, A^{2} \Pi_{u}, B^{2} \Sigma_{u}^{+}$is approximately $1: 4.3 \times 10^{-2}: 8.6 \times$ $10^{-2}$, respectively. For $\mathrm{N}_{2}^{+}$and $\mathrm{O}_{2}^{+}$[31] cations considered

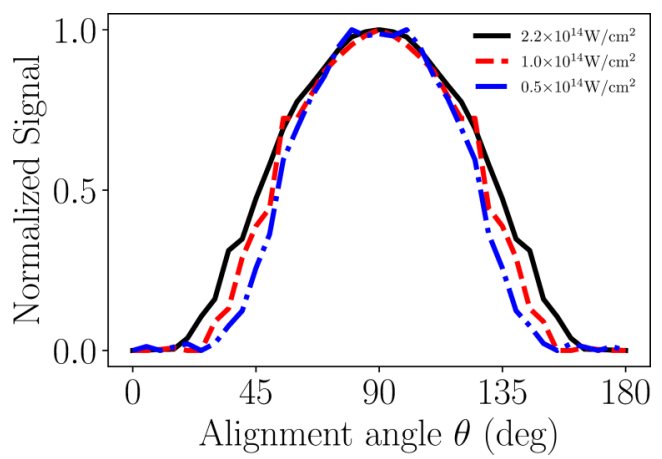

FIG. 6. The angular dependence of dissociation yields of $\mathrm{N}+$ $\mathrm{N}^{+}$when only first order pathway between $X^{2} \Sigma_{g}^{+}$and ${ }^{2} \Pi_{u}$ states exists at various peak laser intensities. 


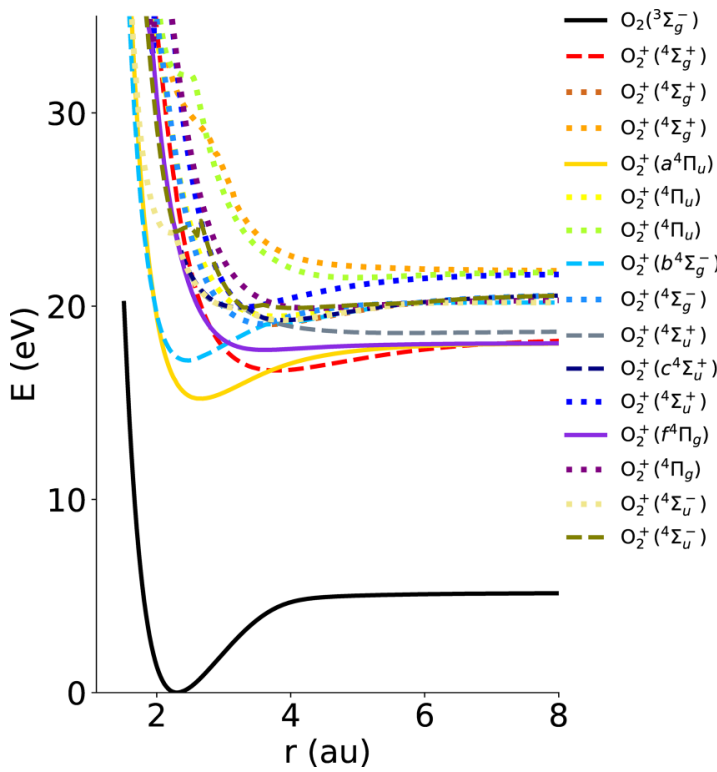

FIG. 7. The potential energy curves of electronic states of $\mathrm{O}_{2}^{+}$ cation. The orange solid line is for the initial excited state $a^{4} \Pi_{u}$, and the purple solid line is for the final dissociative state $f^{4} \Pi_{g}$.

in this work, the single initial state approximation can be a reasonable assumption. In the case that multiple initial states must be taken into account, one has to get the stateresolved dissociation yields from Eq. (1), incorporating the calculated ionization rate $W_{v}$ to different initial state. The energy-integrated and alignment-angle-resolved yields reveal that $D(\theta, \varepsilon)$ maximizes at $\theta=\pi / 2$ and minimizes at $\theta=0$ and $\pi$. At $t=0$ the molecular nitrogen ions are generated from neutral nitrogen molecules and populate the low-lying excited states. The population redistribution starts to occur due to the coupling induced by the remaining laser field. As shown in Fig. 5, from 0 to 5 fs most population in $\mathrm{N}_{2}^{+}\left(X^{2} \Sigma_{g}^{+}\right)$ state is transferred to $\mathrm{N}_{2}^{+}\left(A^{2} \Pi_{u}\right)$ state, and the population in the $\mathrm{N}_{2}^{+}\left(A^{2} \Pi_{u}\right)$ state is greatly enhanced, forming population inversion relative to the cationic ground state $X^{2} \Sigma_{g}^{+}$. Meanwhile, certain amount of population is transferred to the dissociative $\mathrm{N}_{2}^{+}\left({ }^{2} \Pi_{u}\right)$ state. The symmetry selection rule permits first order coupling between $\mathrm{N}_{2}^{+}\left(X^{2} \Sigma_{g}^{+}\right)$and $\mathrm{N}_{2}^{+}\left({ }^{2} \Pi_{u}\right)$. In the period between 5 and $10 \mathrm{fs}$, the population of $\mathrm{N}_{2}^{+}\left(X^{2} \Sigma_{g}^{+}\right)$ is transferred mainly through the intermediate states, $B^{2} \Sigma_{u}^{+}$, $C^{2} \Sigma_{u}^{+}, D^{2} \Pi_{g}$ and ${ }^{2} \Phi_{g}$, to the dissociative states via the third order pathways as listed in Table I. Oscillatory population exchange between the $X^{2} \Sigma_{g}^{+}$and $A^{2} \Pi_{u}$ states is originated from Rabi cycles in the laser field. Since the transition dipole moments between $X^{2} \Sigma_{g}^{+}$and ${ }^{2} \Pi_{u}, B^{2} \Sigma_{u}^{+}$and $D^{2} \Pi_{g}, B^{2} \Sigma_{u}^{+}$ and ${ }^{2} \Phi_{g}, C^{2} \Sigma_{u}^{+}$and $D^{2} \Pi_{g}, C^{2} \Sigma_{u}^{+}$and ${ }^{2} \Phi_{g}$ are perpendicular to the molecular axis, the projection of the electric field on the molecular axis amounts to an effective field strength $E_{\text {eff } \perp}=E_{0} \sin \theta$, where $E_{0} \propto \sqrt{I_{0}}$ is the peak field strength of the ionization pulse. The transition-dipole moment between $X^{2} \Sigma_{g}^{+}$and $B^{2} \Sigma_{u}^{+}, X^{2} \Sigma_{g}^{+}$and $C^{2} \Sigma_{u}^{+}, D^{2} \Pi_{g}$ and ${ }^{2} \Pi_{u},{ }^{2} \Phi_{g}$ and ${ }^{2} \Pi_{u}$ is parallel to the molecular axis, and the projection of the electric field on the molecular axis amounts to an effective field strength $E_{\text {eff } \|}=E_{0} \cos \theta$. Thus the angular dependence of the third order transitions in $E_{\text {eff } \perp}$ and in $E_{\text {eff } \|}$ translates into a spectrum structure for $\theta$ between 0 and $\pi$. The analysis above suggests that the intensity dependence of alignment-angle-resolved yields contains information about the population dynamics and can be used to quantitatively analyze the pathways of PPRM.

After $\sim 50 \mathrm{fs}$, the population reaches equilibrium because the laser coupling disappears. The simulation shows that the
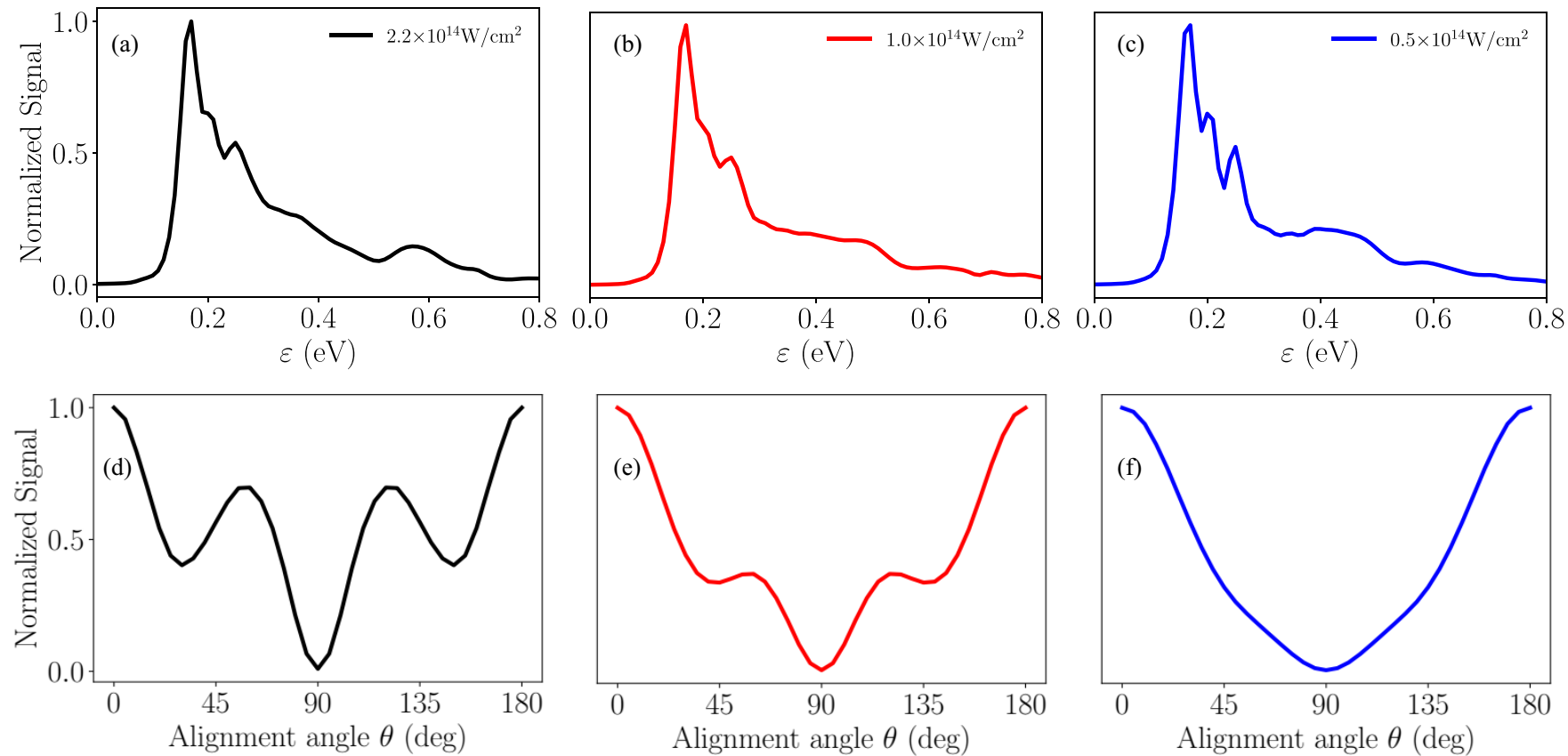

FIG. 8. Kinetic energy and alignment angle dependence of $\mathrm{O}_{2}^{+}$dissociation induced by strong field ionization. The upper panels (a)-(c) are alignment angle-integrated energy distributions. The lower panel (d)-(f) are energy-integrated angular distributions. From left to right columns, the intensity of laser is $2.2 \times 10^{14} \mathrm{~W} / \mathrm{cm}^{2}, 1.0 \times 10^{14} \mathrm{~W} / \mathrm{cm}^{2}$, and $0.5 \times 10^{14} \mathrm{~W} / \mathrm{cm}^{2}$, respectively. 

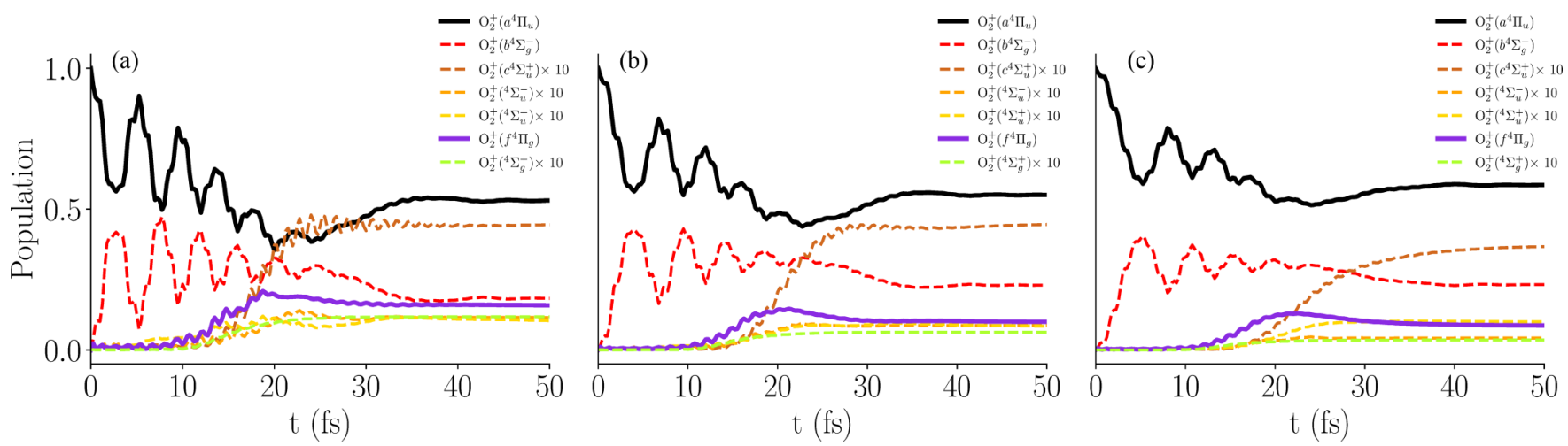

FIG. 9. Temporal population evolution of $a^{4} \Pi_{u}, b^{4} \Sigma_{g}^{-}, c^{4} \Sigma_{u}^{+},{ }^{4} \Sigma_{u}^{-},{ }^{4} \Sigma_{u}^{+}, f^{4} \Pi_{g}$, and ${ }^{4} \Sigma_{g}^{+}$states of $\mathrm{O}_{2}^{+}$cation under different ionization laser intensity at alignment angle $\theta=60$ degrees. From left to right, the peak laser intensity is $2.2 \times 10^{14} \mathrm{~W} / \mathrm{cm}^{2}, 1.0 \times 10^{14} \mathrm{~W} / \mathrm{cm}^{2}$, and $0.5 \times 10^{14} \mathrm{~W} / \mathrm{cm}^{2}$, respectively.

$\mathrm{N}_{2}^{+}$dissociation is dominated by the ${ }^{2} \Pi_{u}$ state, and the wave packet reaches the flux plane at $\sim 70 \mathrm{fs}$. The ${ }^{2} \Phi_{u}$ state also provides minor contribution to dissociation, which does not exceed $10 \%$ at highest pulse intensity in our study.

Next we examine the interpretation of alignment-angleresolved yields based on PPRM for various ionization pulse intensities. As shown in Figs. 4(d)-4(f), the alignment angleresolved yields exhibit apparent broadening as the peak intensity of ionization pulse increases.

It should be noted that although there is direct coupling between the $\mathrm{N}_{2}^{+}\left(X^{2} \Sigma_{g}^{+}\right)$and dissociative ${ }^{2} \Pi_{u}$ states, the participation of the intermediate states $\mathrm{N}_{2}^{+}\left(B^{2} \Sigma_{u}^{+}, C^{2} \Sigma_{u}^{+}, D^{2} \Pi_{g}\right.$, and ${ }^{2} \Phi_{g}$ ) is indispensable for generating the observed intensity dependent angular distribution at the non-perpendicular angle. As evidenced by Fig. 6, if we remove the coupling between $\mathrm{N}_{2}^{+}\left(X^{2} \Sigma_{g}^{+}\right)$and intermediate states $\left(B^{2} \Sigma_{u}^{+}, C^{2} \Sigma_{u}^{+}\right.$, $D^{2} \Pi_{g}$, and ${ }^{2} \Phi_{g}$ ), dissociated states ${ }^{2} \Pi_{u}$ and those intermediate states, the dissociation will dominantly take place for $\theta=\pi / 2$ and exhibit almost no intensity dependence.

\section{B. Ionization and dissociation of $\mathrm{O}_{2}^{+}$cation}

Similarly, the potential energy curves of neutral and cationic oxygen molecule are shown in Fig. 7 [49,50], and $a^{4} \Pi_{u}$ state is dominantly populated when $\mathrm{O}_{2}$ is ionized via strong field tunneling mechanism [8,31]. In Fig. 8, we present the normalized alignment-angle-integrated yields for ionization pulse with peak intensities of $2.2 \times 10^{14} \mathrm{~W} / \mathrm{cm}^{2}, 1.0 \times$ $10^{14} \mathrm{~W} / \mathrm{cm}^{2}$, and $0.5 \times 10^{14} \mathrm{~W} / \mathrm{cm}^{2}$, respectively. The peak at $\sim 0.17 \mathrm{eV}$ is solely contributed from dissociation in $f^{4} \Pi_{g}$ state, which is excited from $a^{4} \Pi_{u}$ (see Fig. 7). Moreover, the kinetic-energy-integrated angle-resolved yields are presented in Fig. 8. The laser intensity dependent angular distribution of dissociation yields of $\mathrm{O}_{2}^{+}$can be similarly explained with the different pathways of PPRM. The $\mathrm{O}_{2}^{+}$dissociation dominantly takes place on the $f^{4} \Pi_{g}$ state, when the initial wave packet is launched in the $a^{4} \Pi_{u}$ state by strong field ionization [8].

In the MCTDH simulation, we assume that the cation is generated from neutral oxygen molecules at $t=0$ and dominantly populates the excited state $\mathrm{O}_{2}^{+}\left(a^{4} \Pi_{u}\right)$. During the time period between 0 and $15 \mathrm{fs}$, most population in $\mathrm{O}_{2}^{+}\left(a^{4} \Pi_{u}\right)$ state is transferred to $\mathrm{O}_{2}^{+}\left(b^{4} \Sigma_{g}^{-}\right)$stateas shown in Fig. 9, and Rabi cycles exist between $a^{4} \Pi_{u}$ and $b^{4} \Sigma_{g}^{-}$ states. In the period between 15 and $50 \mathrm{fs}$, the population of $\mathrm{O}_{2}^{+}\left(a^{4} \Pi_{u}\right)$ is transferred to the dissociative state $\mathrm{O}_{2}^{+}\left(f^{4} \Pi_{g}\right)$ via first order laser-induced coupling and via intermediate states $b^{4} \Sigma_{g}^{-}, c^{4} \Sigma_{u}^{+},{ }^{4} \Sigma_{u}^{-},{ }^{4} \Sigma_{u}^{+}$, and ${ }^{4} \Sigma_{g}^{+}$due to the thirdorder laser-induced coupling, as listed in Table II. Since the transition-dipole moments between $a^{4} \Pi_{u}$ and ${ }^{4} \Sigma_{g}^{+}, a^{4} \Pi_{u}$ and $b^{4} \Sigma_{g}^{-},{ }^{4} \Sigma_{u}^{+}$and $f^{4} \Pi_{g}, c^{4} \Sigma_{u}^{+}$and $f^{4} \Pi_{g},{ }^{4} \Sigma_{u}^{-}$and $f^{4} \Pi_{g}$ are perpendicular to the molecular axis, the projection of the electric field on the molecular axis amounts to an effective field strength $E_{\text {eff } \perp}=E_{0} \sin \theta$. The transition dipole moments between $a^{4} \Pi_{u}$ and $f^{4} \Pi_{g},{ }^{4} \Sigma_{g}^{+}$and ${ }^{4} \Sigma_{u}^{+},{ }^{4} \Sigma_{g}^{+}$and $c^{4} \Sigma_{u}^{+}$, $b^{4} \Sigma_{g}^{-}$and ${ }^{4} \Sigma_{u}^{-}$are parallel to the molecular axis, and the projection of the electric field on the molecular axis amounts to an effective field strength $E_{\text {eff } \|}=E_{0} \cos \theta$. The $\theta$ dependence of the third order transition in $E_{\text {eff } \perp}$ and in $E_{\text {eff } \|}$ translates into variation of angular distribution of fragments between $\theta=0$ and $\pi$. This fact suggests that the angular distribution of alignment-angle-resolved yields contains information about the pathways to reach the dissociative final states, and from which we could classify the pathways of population transfer by their orders. For time after $50 \mathrm{fs}$, the population of mainly populated dissociative state $f^{4} \Pi_{g}$ slowly decreases as the wave packet reaches the flux plane, and in the calculation, the $f^{4} \Pi_{g}$ state contributes to over $97 \%$ of the total dissociation yield.

We then examine the PPRM interpretation in alignmentangle-resolved yields for weaker pulse intensities. For this purpose, we show angle-integrated and energy-integrated

TABLE II. The first and third order pathways for population transfer in $\mathrm{O}_{2}^{+}$cation, and their dependence on alignment angle and electronic field strength.

\begin{tabular}{lc}
\hline \hline Channel & $(\theta, E)$-dependence \\
\hline$f^{4} \Pi_{g} \stackrel{\mu_{\|}}{\longleftarrow} a^{4} \Pi_{u}$ & $E \cos \theta$ \\
$f^{4} \Pi_{g} \stackrel{\mu_{\perp}}{\longleftarrow}{ }^{4} \Sigma_{u}^{+} \stackrel{\mu_{\|}}{{ }^{4}}{ }^{4} \Sigma_{g}^{+} \stackrel{\mu_{\perp}}{\longleftarrow} a^{4} \Pi_{u}$ & $E^{3} \cos \theta \sin ^{2} \theta$ \\
$f^{4} \Pi_{g} \stackrel{\mu_{\perp}}{\longleftarrow} c^{4} \Sigma_{u}^{+} \stackrel{\mu_{\|}}{{ }^{4} \Sigma_{g}^{+} \stackrel{\mu_{\perp}}{\longleftarrow} a^{4} \Pi_{u}}$ & $E^{3} \cos \theta \sin ^{2} \theta$ \\
$f^{4} \Pi_{g} \stackrel{\mu_{\perp}}{\longleftarrow}{ }^{4} \Sigma_{u}^{-} \stackrel{\mu_{\|}}{\longleftarrow} b^{4} \Sigma_{g}^{-} \stackrel{\mu_{\perp}}{\longleftarrow} a^{4} \Pi_{u}$ & $E^{3} \cos \theta \sin ^{2} \theta$ \\
\hline \hline
\end{tabular}




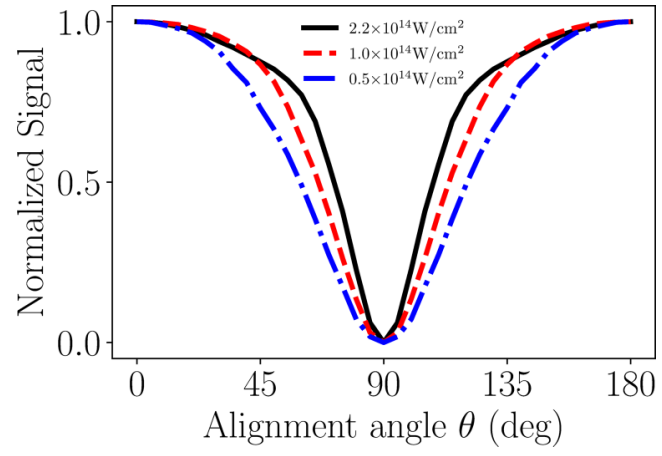

FIG. 10. The alignment angle dependence of dissociation yields of $\mathrm{O}+\mathrm{O}^{+}$under various laser intensities, assuming that only first order pathway is present. Black solid line, red dashed line and blue dot dashed line denote the distributions at peak laser intensities of $2.2 \times 10^{14} \mathrm{~W} / \mathrm{cm}^{2}$, of $1.0 \times 10^{14} \mathrm{~W} / \mathrm{cm}^{2}$, and of $0.5 \times 10^{14} \mathrm{~W} / \mathrm{cm}^{2}$, respectively.

yields for pulse peak intensities of $2.2 \times 10^{14} \mathrm{~W} / \mathrm{cm}^{2}$, $1.0 \times 10^{14} \mathrm{~W} / \mathrm{cm}^{2}$, and $0.5 \times 10^{14} \mathrm{~W} / \mathrm{cm}^{2}$ in Fig. 8, respectively. The calculated angle-resolved yields exhibit intensitydependent angular distribution. As the peak intensity of ionization laser increases, the amplitudes of third order processes become stronger than that at $0.5 \times 10^{14} \mathrm{~W} / \mathrm{cm}^{2}$, and the oscillatory behavior is smeared out in $D(\theta, \varepsilon)$, as the transitions to intermediate $\mathrm{O}_{2}^{+}$states $b^{4} \Sigma_{g}^{-}, c^{4} \Sigma_{u}^{+},{ }^{4} \Sigma_{u}^{-},{ }^{4} \Sigma_{u}^{+}$ and ${ }^{4} \Sigma_{g}^{+}$apparently interfere. As evidenced by Fig. 10, if we remove the coupling between $\mathrm{O}_{2}^{+}\left(a^{4} \Pi_{u}, f^{4} \Pi_{g}\right)$ and intermediate states $\left(b^{4} \Sigma_{g}^{-}, c^{4} \Sigma_{u}^{+},{ }^{4} \Sigma_{u}^{-},{ }^{4} \Sigma_{u}^{+}\right.$, and $\left.{ }^{4} \Sigma_{g}^{+}\right)$, the dissociation will dominantly take place in parallel alignment, and the oscillatory to smooth transition in the alignment-angle-dependent yield will not occur.

\section{BRANCHING RATIO OF PPRM PATHWAYS}

Using symmetry selection rules listed in Table I and Table II, the transitions from cationic ground states to final dissociative states involve only odd order processes. Since the highest laser intensity in our investigation is $2.2 \times$ $10^{14} \mathrm{~W} / \mathrm{cm}^{2}$, which corresponds to the field strength of 0.079 a.u., processes higher than third order are neglected. As shown in Fig. 11, the model consisting of first and third order transitions excellently reproduces the variation of dissociation yields as a function of ionization laser intensity for both $\mathrm{N}_{2}^{+}$and $\mathrm{O}_{2}^{+}$cation. In the model, we fit the alignmentangle-dependent dissociation yields for $\mathrm{N}_{2}^{+}$and $\mathrm{O}_{2}^{+}$using the following equations:

$$
\begin{aligned}
D_{\mathrm{N}_{2}^{+}}(\theta)= & A_{0 \mathrm{M}}^{2} \sin ^{2} \theta+B_{2 \mathrm{M}}^{2} \sin ^{2} \theta \cos ^{4} \theta \\
& +2 A_{0 \mathrm{M}} B_{2 \mathrm{M}} C \sin ^{2} \theta \cos ^{2} \theta, \\
D_{\mathrm{O}_{2}^{+}}(\theta)= & A_{0 \mathrm{M}}^{2} \cos ^{2} \theta+B_{2 \mathrm{M}}^{2} \cos ^{2} \theta \sin ^{4} \theta \\
& +2 A_{0 \mathrm{M}} B_{2 \mathrm{M}} C \sin ^{2} \theta \cos ^{2} \theta,
\end{aligned}
$$

where $A_{0 M}, B_{2 M}$ are transition amplitudes of first and third order transitions with 0 and 2 intermediate states, respectively. $C=\cos \phi$ represents the effective phase difference $\phi$ between two types of channels. For $\mathrm{N}_{2}^{+}$cation, though transitions from initial $X^{2} \Sigma_{g}^{+}$state to the final ${ }^{2} \Phi_{u}$ state could also provide minor dissociation yield $(<0.1$ under highest laser intensity), it has the same alignment angle-dependence as the transitions between $X^{2} \Sigma_{g}^{+}$and ${ }^{2} \Pi_{u}$ states, i.e., the amplitudes are proportional to $E \sin \theta$ for first order transition and proportional to $E^{3} \cos ^{2} \theta \sin \theta$ for third order transitions.

The branching ratio of first and third order channels for $\mathrm{N}_{2}^{+}$and $\mathrm{O}_{2}^{+}$is shown in Table III and Table IV, respectively. As expected, the branching ratio of third order channels greatly increases at higher laser intensities, which
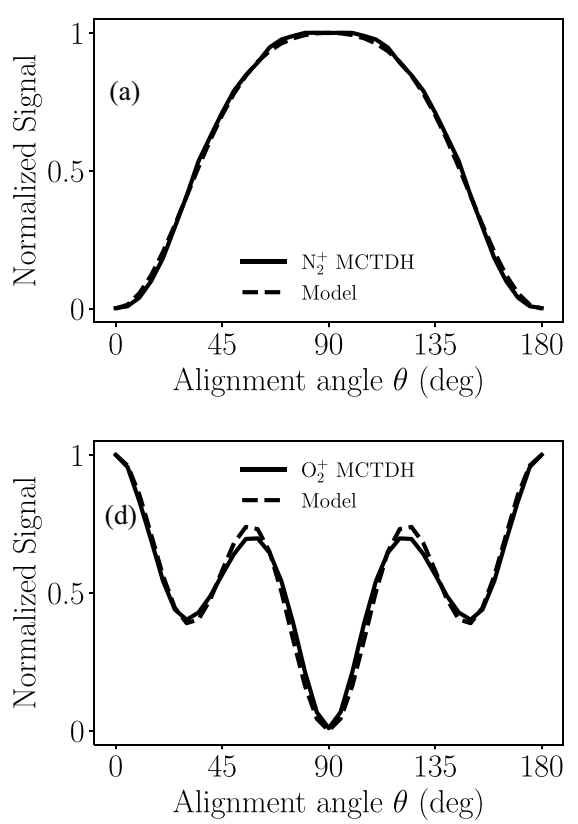
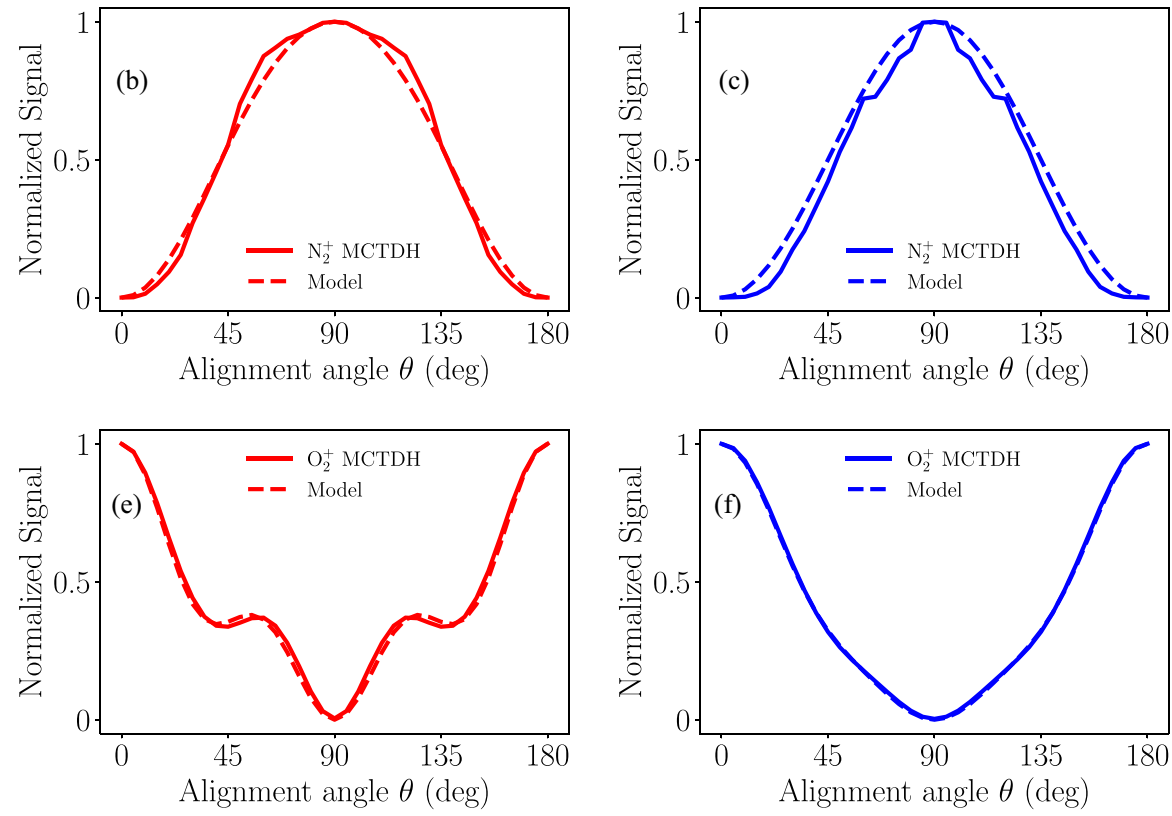

FIG. 11. The upper panel shows the comparison of $\mathrm{N}_{2}^{+}$dissociation yields between MCTDH simulation and the model in Eq. (14) for peak laser intensities $2.2 \times 10^{14} \mathrm{~W} / \mathrm{cm}^{2}, 1.0 \times 10^{14} \mathrm{~W} / \mathrm{cm}^{2}$, and $0.5 \times 10^{14} \mathrm{~W} / \mathrm{cm}^{2}$, respectively. The lower panel shows the comparison of $\mathrm{O}_{2}^{+}$ dissociation for the same set of intensities. Solid and dashed lines indicate the results of MCTDH simulation and the model, respectively. 
TABLE III. The branching ratio of first and third order pathways of $\mathrm{N}_{2}^{+}$under three ionization laser intensities.

\begin{tabular}{lcc}
\hline \hline & \multicolumn{2}{c}{ Branching ratio } \\
\cline { 2 - 3 } Peak laser intensity $\left(10^{14} \mathrm{~W} / \mathrm{cm}^{2}\right)$ & $\frac{A_{0 \mathrm{M}}^{2}}{A_{0 \mathrm{M}}^{2}+B_{2 \mathrm{M}}^{2}}$ & $\frac{B_{2 \mathrm{M}}^{2}}{A_{0 \mathrm{M}}^{2}+B_{2 \mathrm{M}}^{2}}$ \\
\hline 2.2 & 0.880 & 0.120 \\
1.0 & 0.990 & 0.010 \\
0.5 & 1.000 & 0.000 \\
\hline \hline
\end{tabular}

indicates that PPRM affects the dissociation process by multilevel transitions involving participation of intermediate states. Because the non-Born-Oppenheimer radial coupling between the electronic states are not taken into account, the dissociative states could not receive transferred population after the laser pulse, and the total dissociation ratio could be underestimated. However, the nonadiabatic radial coupling does not depend on the alignment angle and its contribution will not affect the analysis of dissociative ionization pathways. Note that the rotational-coupling is generally much smaller than the radial-coupling and can be safely ignored.

\section{CONCLUSION}

We have performed theoretical investigations for the post-ionization population redistribution and dissociation dynamics of $\mathrm{N}_{2}$ and $\mathrm{O}_{2}$ molecules with intense short ionization laser pulse. We calculated the alignment-angle-resolved kinetic energy release spectra of the dissociated fragments and the variation as a function of ionization laser intensity, from which the branching ratio of first and third order population transfer processes can be determined. Our method can be conveniently applied in analysis of realistic experiments of
TABLE IV. The branching ratio of first and third order pathways of $\mathrm{O}_{2}^{+}$under three ionization laser intensities.

\begin{tabular}{lcc}
\hline \hline & \multicolumn{2}{c}{ Branching ratio } \\
\cline { 2 - 3 } Peak laser intensity $\left(10^{14} \mathrm{~W} / \mathrm{cm}^{2}\right)$ & $\frac{A_{0 \mathrm{M}}^{2}}{A_{0 \mathrm{M}}^{2}+B_{2 \mathrm{M}}^{2}}$ & $\frac{B_{2 \mathrm{M}}^{2}}{A_{0 \mathrm{M}}^{2}+B_{2 \mathrm{M}}^{2}}$ \\
\hline 2.2 & 0.100 & 0.900 \\
1.0 & 0.174 & 0.826 \\
0.5 & 0.442 & 0.558 \\
\hline \hline
\end{tabular}

strong field dissociative ionization of molecules, and more generally, in analyzing ultrafast population dynamics induced by laser-molecule interaction. Though we have not addressed the population transfer pathways to the final bound states of the monocations in direct dissociative ionization process, our method can be generalized to analyze the pathways to these bound states as well following the same principle, e.g., by introducing an ionization pulse that excites a final bound state to a dissociative or predissociative state [51]. We could apply the analysis in this work to the resulting dissociation yields. Theoretical schemes to fully disentangle individual population transfer pathways in systems consisting of multiple initial and final states are being pursued.

\section{ACKNOWLEDGMENTS}

The authors are thankful to Hans-Dieter Meyer, Oriol Vendrell, and Yunquan Liu for helpful discussions. This work is supported by National Key Research and Development Program of China (Grant Nos. 2019YFA0308300 and 2020YFA0211300) and National Natural Science Foundation of China (Grant Nos. 11722432, 12021004, 92050201, 11974031, and 61475055).
[1] W. T. Hill III and C. H. Lee, Light-Matter Interaction: Atoms and Molecules in External Fields and Nonlinear Optics (WileyVCH, New York, 2007).

[2] F. Großmann, Theoretical Femtosecond Physics: Atoms and Molecules in Strong Laser Fields (Springer Verlag, Berlin, 2008).

[3] Q. Zhang et al., Sub-cycle coherent control of ionic dynamics via transient ionization injection, Commun. Phys. 3, 50 (2020).

[4] S. Li, D. Sierra-Costa, M. J. Michie, I. Ben-Itzhak, and M. Dantus, Control of electron recollision and molecular nonsequential double ionization, Commun. Phys. 3, 35 (2020).

[5] E. Goulielmakis et al., Real-time observation of valence electron motion, Nature (London) 466, 739 (2010).

[6] M. Ossiander et al., Attosecond correlation dynamics, Nat. Phys. 13, 280 (2017).

[7] M. Sabbar et al., State-resolved attosecond reversible and irreversible dynamics in strong optical fields, Nat. Phys. 13, 472 (2017).

[8] P. Cörlin, A. Fischer, M. Schönwald, A. Sperl, T. Mizuno, U. Thumm, T. Pfeifer, and R. Moshammer, Probing calculated $\mathrm{O}_{2}{ }^{+}$ potential-energy curves with an XUV-IR pump-probe experiment, Phys. Rev. A 91, 043415 (2015).
[9] D. A. Card, E. S. Wisniewski, D. E. Folmer, and A. W. Castleman, Dynamics of Coulomb explosion and kinetic energy release in clusters of heterocyclic compounds, J. Chem. Phys. 116, 3554 (2002).

[10] D. M. Bittner, K. Gope, and D. Strasser, Time-resolved dissociative ionization and double photoionization of $\mathrm{CO}_{2}$, J. Chem. Phys. 153, 194201 (2020).

[11] F. Allum et al., Coulomb explosion imaging of $\mathrm{CH}_{3} \mathrm{I}$ and $\mathrm{CH}_{2} \mathrm{ClI}$ photodissociation dynamics, J. Chem. Phys. 149, 204313 (2018).

[12] I. Last and J. Jortner, Electron and nuclear dynamics of molecular clusters in ultraintense laser fields. IV. Coulomb explosion of molecular heteroclusters, J. Chem. Phys. 121, 8329 (2004).

[13] C. Wu et al., Three-body fragmentation of $\mathrm{CO}_{2}$ driven by intense laser pulses, J. Chem. Phys. 142, 124303 (2015).

[14] A. Giusti-Suzor, F. H. Mies, L. F. DiMauro, E. Charron, and B. Yang, Dynamics of $\mathrm{H}_{2}^{+}$in intense laser fields, J. Phys. B: At. Mol. Opt. Phys. 28, 309 (1995).

[15] P. H. Bucksbaum, A. Zavriyev, H. G. Muller, and D. W. Schumacher, Softening of the $\mathrm{H}_{2}^{+}$Molecular Bond in Intense Laser Fields, Phys. Rev. Lett. 64, 1883 (1990). 
[16] A. Giusti-Suzor, X. He, O. Atabek, and F. H. Mies, Abovethreshold dissociation of $\mathrm{H}_{2}^{+}$in intense laser fields, Phys. Rev. Lett. 64, 515 (1990).

[17] E. Charron, A. Giusti-Suzor, and F. H. Mies, Two-color coherent control of $\mathrm{H}_{2}^{+}$photodissociation in intense laser fields, Phys. Rev. Lett. 71, 692 (1993).

[18] K. Sändig, H. Figger, and T. W. Hänsch, Dissociation Dynamics of $\mathrm{H}_{2}^{+}$in Intense Laser Fields: Investigation of Photofragments from Single Vibrational Levels, Phys. Rev. Lett. 85, 4876 (2000).

[19] X. Urbain, B. Fabre, E. M. Staicu-Casagrande, N. deRuette, V. M. Andrianarijaona, J. Jureta, J. H. Posthumus, A. Saenz, E. Baldit, and C. Cornaggia, Intense-Laser-Field Ionization of Molecular Hydrogen in the Tunneling Regime and Its Effect on the Vibrational Excitation of $\mathrm{H}_{2}^{+}$, Phys. Rev. Lett. 92, 163004 (2004).

[20] T. Ergler, A. Rudenko, B. Feuerstein, K. Zrost, C. D. Schroter, R. Moshammer, and J. Ullrich, Time-Resolved Imaging and Manipulation of $\mathrm{H}_{2}$ Fragmentation in Intense Laser Fields, Phys. Rev. Lett. 95, 093001 (2005).

[21] T. Ergler, A. Rudenko, B. Feuerstein, K. Zrost, C. D. Schroter, R. Moshammer, and J. Ullrich, Spatiotemporal Imaging of Ultrafast Molecular Motion: Collapse and Revival of the $\mathrm{D}_{2}^{+}$ Nuclear Wave Packet, Phys. Rev. Lett. 97, 193001 (2006).

[22] M. F. Kling et al., Control of electron localization in molecular dissociation, Science 312, 246 (2006).

[23] D. Ray, F. He, S. De, W. Cao, H. Mashiko, P. Ranitovic, K. P. Singh, I. Znakovskaya, U. Thumm, G. G. Paulus, M. F. Kling, I. V. Litvinyuk, and C. L. Cocke, Ion-Energy Dependence of Asymmetric Dissociation of $\mathrm{D}_{2}$ by a Two-Color Laser Field, Phys. Rev. Lett. 103, 223201 (2009).

[24] J. Wu, M. Kunitski, M. Pitzer, F. Trinter, L. P. H. Schmidt, T. Jahnke, M. Magrakvelidze, C. B. Madsen, L. B. Madsen, U. Thumm, and R. Dorner, Electron-Nuclear Energy Sharing in Above-Threshold Multiphoton Dissociative Ionization of $\mathrm{H}_{2}^{+}$, Phys. Rev. Lett. 111, 023002 (2013).

[25] M. Odenweller, N. Takemoto, A. Vredenborg, K. Cole, K. Pahl, J. Titze, L. P. H. Schmidt, T. Jahnke, R. Dorner, and A. Becker, Strong Field Electron Emission from Fixed in Space $\mathrm{H}_{2}^{+}$Ions, Phys. Rev. Lett. 107, 143004 (2011).

[26] A. Rudenko et al., Fragmentation dynamics of molecular hydrogen in strong ultrashort laser pulses, J. Phys. B: At. Mol. Opt. Phys. 38, 487 (2005).

[27] R. Numico, A. Keller, and O. Atabek, Intense-laser-induced alignment in angularly resolved photofragment distributions of $\mathrm{H}_{2}^{+}$, Phys. Rev. A 60, 406 (1999).

[28] V. N. Serov, A. Keller, O. Atabek, and N. Billy, Quantitative theory-versus-experiment comparison for the intense laser dissociation of $\mathrm{H}_{2}^{+}$, Phys. Rev. A 68, 053401 (2003).

[29] S. Pabst, L. Greenman, P. J. Ho, D. A. Mazziotti, and R. Santra, Decoherence in Attosecond Photoionization, Phys. Rev. Lett. 106, 053003 (2011).

[30] X. M. Tong, Z. X. Zhao, and C. D. Lin, Theory of molecular tunneling ionization, Phys. Rev. A 66, 033402 (2002).

[31] S. F. Zhao, C. Jin, A.-T. Le, T. F. Jiang, and C. D. Lin, Determination of structure parameters in strong-field tunneling ionization theory of molecules, Phys. Rev. A 81, 033423 (2010).

[32] S. B. Zhang and N. Rohringer, Photoemission spectroscopy with high-intensity short-wavelength lasers, Phys. Rev. A 89, 013407 (2014).
[33] H. Xu, E. Lötstedt, A. Iwasaki, and K. Yamanouchi, Sub-10-fs population inversion in $\mathrm{N}_{2}^{+}$in air lasing through multiple state coupling, Nat. Commun. 6, 8347 (2015).

[34] P. A. Carpeggiani et al., Complete reconstruction of bound and unbound electronic wavefunctions in two-photon double ionization, Nat. Phys. 15, 170 (2019).

[35] H. Li, M. Hou, H. Zang, Y. Fu, E. Lotstedt, T. Ando, A. Iwasaki, K. Yamanouchi, and H. Xu, Significant Enhancement of $\mathrm{N}_{2}^{+}$Lasing by Polarization-Modulated Ultrashort Laser Pulses, Phys. Rev. Lett. 122, 013202 (2019).

[36] H. Li, E. Lotstedt, H. Li, Y. Zhou, N. Dong, L. Deng, P. Lu, T. Ando, A. Iwasaki, Y. Fu, S. Wang, J. Wu, K. Yamanouchi, and $\mathrm{H}$. Xu, Giant Enhancement of Air Lasing by Complete Population Inversion in $\mathrm{N}_{2}^{+}$, Phys. Rev. Lett. 125, 053201 (2020).

[37] J. Yao, S. Jiang, W. Chu, B. Zeng, C. Wu, R. Lu, Z. Li, H. Xie, G. Li, C. Yu, Z. Wang, H. Jiang, Q. Gong, and Y. Cheng, Population Redistribution Among Multiple Electronic States of Molecular Nitrogen Ions in Strong Laser Fields, Phys. Rev. Lett. 116, 143007 (2016).

[38] P. M. Abanador and U. Thumm, Characterization of lightinduced potentials in the strong-field dissociation of $\mathrm{O}_{2}^{+}$, Phys. Rev. A 102, 053114 (2020).

[39] S.-F. Zhao, A.-T. Le, C. Jin, X. Wang, and C. D. Lin, Analytical model for calibrating laser intensity in strong-field-ionization experiments, Phys. Rev. A 93, 023413 (2016).

[40] M. Beck, The multiconfiguration time-dependent Hartree (MCTDH) method: A highly efficient algorithm for propagating wavepackets, Phys. Rep. 324, 1 (2000).

[41] G. A. Worth, M. H. Beck, A. Jäckle, and H.-D. Meyer, The MCTDH package, version 8.2 (2000); H.-D. Meyer, version 8.3 (2002); version 8.4 (2007); see http://mctdh.uni-hd.de.

[42] A. Jäckle and H. Meyer, Time-dependent calculation of reactive flux employing complex absorbing potentials: General aspects and application within the multiconfiguration timedependent Hartree wave approach, J. Chem. Phys. 105, 6778 (1996).

[43] Y.-Z. Fu, S.-F. Zhao, and X.-X. Zhou, Multiphoton and tunneling ionization of atoms in an intense laser field, Chinese Phys. B 21, 113101 (2012).

[44] H.-J. Werner et al., MOLPRO, version 2015.1, a package of $a b$ initio programs (2015), see https://www.molpro.net.

[45] X. Li and J. Paldus, Full potential energy curve for $\mathrm{N}_{2}$ by the reduced multireference coupled-cluster method, J. Chem. Phys. 129, 054104 (2008).

[46] R. J. Le Roy, Y. Huang, and C. Jary, An accurate analytic potential function for ground-state $\mathrm{N}_{2}$ from a direct-potential-fit analysis of spectroscopic data, J. Chem. Phys. 125, 164310 (2006).

[47] C. Stemmle, C. J. Villabona, C. Forero, B. Paulus, and J. F. Pérez-Torres, Isotopic and temperature effects on the photoemission and predissociation rates of $\mathrm{N}_{2}^{+}\left(C^{2} \Sigma_{u}^{+}\right)$, Phys. Rev. A 102, 032802 (2020).

[48] D. T. Colbert and W. H. Miller, A novel discrete variable representation for quantum mechanical reactive scattering via the S-matrix Kohn method, J. Chem. Phys. 96, 1982 (1992).

[49] L. Bytautas, N. Matsunaga, and K. Ruedenberg, Accurate $a b$ initio potential energy curve of $\mathrm{O}_{2}$. II. Core-valence correlations, relativistic contributions, and vibration-rotation spectrum, J. Chem. Phys. 132, 074307 (2010). 
[50] D. Sundholm, J. Olsen, and S. A. Alexander, Finite-element multiconfiguration Hartree-Fock calculations on the excitation energies and the ionization potential of oxygen, J. Chem. Phys. 96, 5229 (1992).
[51] H. Timmers, Z. Li, N. Shivaram, R. Santra, O. Vendrell, and A. Sandhu, Coherent Electron Hole Dynamics Near a Conical Intersection, Phys. Rev. Lett. 113, 113003 (2014). 